\title{
Serum S100B and LDH at Baseline and During Therapy Predict the Outcome of Metastatic Melanoma Patients Treated with BRAF Inhibitors
}

\author{
Maximilian Gassenmaier ${ }^{1} \cdot$ Max M. Lenders ${ }^{1} \cdot$ Andrea Forschner $^{1} \cdot$ Ulrike Leiter $^{1} \cdot$ Benjamin Weide $^{1} \cdot$ Claus Garbe $^{1}$. \\ Thomas K. Eigentler ${ }^{1}$. Nikolaus B. Wagner ${ }^{1,2}$ (D)
}

Accepted: 16 January 2021 / Published online: 8 February 2021

(c) The Author(s) 2021

\begin{abstract}
Background Despite impressive response rates, most patients with advanced melanoma ultimately progress following therapy with B-Raf proto-oncogene (BRAF) inhibitors (BRAFi). Therefore, frequent radiologic assessments are necessary, and reliable serum biomarkers would be beneficial for disease monitoring.

Objective This study investigated the ability of lactate dehydrogenase (LDH) and S100 calcium-binding protein B (S100B) to detect response and disease progression during treatment with BRAFi.

Patients and Methods Baseline levels of LDH and S100B and repeated measurements during therapy were recorded retrospectively in 191 patients with metastatic melanoma. LDH and S100B levels were compared between distinct time points (baseline, first follow-up visit [FV], best objective response [BR], and progressive disease [PD]). The prognostic ability of the serum biomarkers in relation to disease-specific survival (DSS) was assessed with univariable and multivariable Cox regression analysis.

Results Elevated baseline LDH and S100B correlated with impaired DSS. In contrast with LDH $(P=0.12), \mathrm{S} 100 \mathrm{~B}$ levels at FV correlated with response $(P=0.0030)$. Both markers significantly decreased during the first weeks of BRAFi treatment (LDH, $P=0.00034$; S100B, $P<0.0001)$ and increased between BR and PD (LDH, $P=0.016$; S100B, $P<0.0001$ ). Patients with elevated S100B $(P=0.00062)$ but not with elevated LDH $(P=0.067)$ at the time point of radiologically confirmed PD showed significantly impaired DSS after PD. Interestingly, DSS after PD differed significantly according to S100B levels determined as early as 8 weeks (median) before PD $(P=0.0024)$.

Conclusions $\mathrm{LDH}$ and S100B are suitable serum biomarkers during therapy with BRAFi. S100B shows stronger correlation with response and exhibits more accuracy in predicting PD. Close biomarker monitoring with S100B is recommended during treatment with BRAFi to detect PD early.
\end{abstract}

Maximilian Gassenmaier and Max M. Lenders contributed equally to this work.

Nikolaus B. Wagner

nikolausbenjamin.wagner@kssg.ch

1 Department of Dermatology, University Hospital Tübingen, Tübingen, Germany

2 Department of Dermatology and Allergology, Kantonsspital St. Gallen, Rorschacherstrasse 95, 9007 St. Gallen, Switzerland

\section{Key Points}

Lactate dehydrogenase (LDH) and S100 calcium-binding protein B (S100B) are suitable serum biomarkers at baseline and during therapy of metastatic melanoma with B-Raf proto-oncogene (BRAF) inhibitors.

S100B shows stronger correlation with response and exhibits more accuracy in the prediction of progressive disease as early as 8 weeks before radiographic evidence of progression. 


\section{Introduction}

B-Raf proto-oncogene (BRAF) inhibitors (BRAFi) in combination with mitogen-activated protein kinase kinase (MEK) inhibitors (MEKi) have elicited remarkable response rates of 64-68\% in patients with advanced melanoma [1-5]. However, secondary resistance occurs in most patients and, although the median duration of progression-free survival (PFS) is comparable with that of the combined immune checkpoint inhibitors (ICIs) ipilimumab plus nivolumab (BRAFi/MEKi, 9.9-13.8 months; ICI, 11.5 months), the 5-year PFS rate with the BRAFi/MEKi combination is nearly two times lower than that of combined ICI (BRAFi/ MEKi, 19\%; ICI, 36\%) [6-9]. Importantly, PFS and overall survival are markedly reduced in patients with extensive disease, evidenced by increased lactate dehydrogenase (LDH), three or more metastatic sites, or M1c stage [9-11]. Disease progression due to secondary resistance can occur suddenly and with fulminant tumor growth or with a subtle change with a mixed response pattern in follow-up computed tomography (CT) scans. As well as careful radiologic observation of patients at high risk of progression, it seems important to explore potential biomarkers that could ideally predict progression even before radiographic evidence. Unlike ICI, targeted therapy with BRAFi and MEKi directly inhibits tumor growth. Therefore, serum biomarkers that are related to tumor growth and tumor cell turnover might be particularly important in these patients. S100 calcium-binding protein B (S100B; also known as S-100) is thought to have higher sensitivity than LDH in detecting locoregional and distant metastasis in high-risk patients with melanoma $[12,13]$. Nevertheless, the prognostic and predictive impact of S100B in patients treated with BRAFi and MEKi remains elusive. So far, only small studies comprising 44 and 18 patients, respectively, have investigated whether LDH and S100B correlate with initial response and later progression under combined BRAFi and MEKi [14, 15]. These studies showed that decreases in S100B correlated with response to treatment but were contradictory as to whether S100B levels rose with disease progression. Therefore, one major aim of the present study was to investigate S100B and LDH at baseline, first follow-up visit (FV), best objective response (BR), and at disease progression (PD) in a large cohort. Moreover, we aimed to investigate whether S100B and LDH serum levels at PD and even before radiologic evidence of PD are predictive for the further course of the disease.

\section{Methods}

\subsection{Patients}

This retrospective single-center analysis included 191 consecutive patients with unresectable American Joint Committee on Cancer (AJCC) (7th edition; 2009) stage III or IV BRAF-mutant (V600E, V600K, and V600R mutations) metastatic melanoma who had started treatment with a BRAFi with or without a MEKi from August 2010 to December 2016. This study was carried out in accordance with the Declaration of Helsinki of 1975 and succeeding amendments. Approval to conduct this study was obtained from the ethics committee of the Medical Faculty of University Tübingen (project No. 257/2018BO2).

\subsection{Data Collection}

Clinical records were examined and the following patient data extracted: age, sex, AJCC clinical staging (7th edition; 2009), prior treatment, BRAF mutational status, first and best objective response, and LDH and S100B at start date of therapy, first FV, timepoint of BR, last blood draw before PD, and timepoint of ascertained PD (Fig. S1 in the electronic supplementary material [ESM]). Routine laboratory workup at all timepoints included blood counts and serologic factors as recommended by clinical trial protocols. FVs also included symptom-focused history taking, whole body skin examination, and palpation of all relevant lymph node groups. Radiologic staging examinations were usually performed utilizing contrast-enhanced CT scan of the neck, chest, abdomen, and pelvis and magnetic resonance imaging (MRI) of the brain. In cases with unclear findings, a positron emission tomography CT scan was performed in most cases or a control CT scan was undertaken in a timely manner. In cases with unclear hepatic lesions, an MRI of the liver was supplemented. The intended interval between staging examinations was 3 months, with earlier investigation if serum LDH or S100B increased. Upper limits of normal for LDH and $\mathrm{S} 100 \mathrm{~B}$ were $250 \mathrm{U} / \mathrm{l}$ and $0.1 \mu \mathrm{g} / \mathrm{l}$, respectively.

\subsection{Statistical Analysis}

Response to treatment was determined by Response Evaluation Criteria in Solid Tumors (RECIST) version 1.1 [16]. Disease-specific survival (DSS) was measured from start of BRAFi therapy to death due to melanoma (event) or last follow-up (censored). DSS after progression (DSSaP) was calculated from radiologically ascertained PD to death due to melanoma or last follow-up. Survival analysis was conducted utilizing the Kaplan-Meier method, univariable logrank test, and multivariable Cox regression. Multivariable 
analyses were performed as complete case analyses, with patients excluded if values were missing. The sensitivity of S100B and LDH in detecting progression was determined by dividing the number of patients with elevated S100B or LDH showing progression by the number of all progressors. Group comparisons with continuous variables were carried out utilizing Wilcoxon's rank-sum test (Mann-Whitney $U$ test). All analyses were carried out with R, version 3.6.1 (The R Foundation for Statistical Computing, Vienna, Austria) and the 'survival' package. All reported tests were twosided, and $P$-values $<0.05$ were considered significant.

\section{Results}

\subsection{Lactate Dehydrogenase (LDH) and S100 Calcium-Binding Protein B (S100B) as Prognostic Baseline Biomarkers}

A total of 191 patients with available baseline S100B and LDH were evaluated. Table 1 details the patient characteristics at baseline. Of the 191 patients, 94 (49.2\%) had elevated baseline $\mathrm{LDH}$, and 133 (69.6\%) had elevated baseline S100B. Elevated baseline levels of both biomarkers were significantly associated with impaired DSS (LDH, hazard ratio [HR] 2.61 [95\% confidence interval $\{\mathrm{CI}\} 1.82-3.76], P$ $<0.0001$; S100B, HR 1.70 [95\% CI 1.14-2.53], $P=0.0096$ ) (Fig. 1).

\subsection{Accuracy of LDH and S100B when Correlated with Best Objective Response and Progression}

Early levels of LDH and S100B at the first follow-up visit (FV) were analyzed according to best objective response achieved during BRAFi treatment (Fig. 2). LDH serum levels at FV did not differ significantly between patients achieving partial response (PR)/complete response (CR) (median 239 U/l; interquartile range [IQR] 199-282]) and those with stable disease (SD) or PD (median 242 U/l; IQR 208-330; $P=0.12$ ) (Fig. 2a). In contrast, S100B levels at FV were significantly lower in patients with PR/CR (median 0.076 $\mu \mathrm{g} / \mathrm{l}$; IQR $0.060-0.118$ ) than in those with SD/PD (median $0.114 \mu \mathrm{g} / \mathrm{l}$; IQR 0.069-0.390; $P=0.0030$ ) (Fig. 2b).

In patients eventually showing PD $(n=121), \mathrm{LDH}$ and S100B were compared between four distinct timepoints: baseline, FV, BR, and PD. Although neither LDH (60/121 [49.6\%]) nor S100B (70/121 [57.9\%]) reached high levels of sensitivity in the prediction of $\mathrm{PD}$, serum levels of both LDH $(P=0.00034)$ and S100B $(P<0.0001)$ decreased significantly from baseline to $\mathrm{FV}$, indicating initial response to treatment (Fig. 3). The median interval between baseline and FV was 36 days (IQR 25-57; data not shown). Comparing the biomarker levels between BR and PD, serum
Table 1 Characteristics of the study population

\begin{tabular}{|c|c|}
\hline Characteristic & $N(\%)$ \\
\hline \multicolumn{2}{|l|}{ Age (years) } \\
\hline$\leq 60$ & $122(63.9)$ \\
\hline$>60$ & $69(36.1)$ \\
\hline \multicolumn{2}{|l|}{ Sex } \\
\hline Female & $85(44.5)$ \\
\hline Male & $106(55.5)$ \\
\hline \multicolumn{2}{|l|}{ BRAF mutational status } \\
\hline V600E & $120(62.8)$ \\
\hline V600K & $15(7.9)$ \\
\hline V600R & $3(1.6)$ \\
\hline V600, not further specified & $53(27.7)$ \\
\hline \multicolumn{2}{|l|}{ Number of sites involved } \\
\hline 1 & $31(16.2)$ \\
\hline 2 & $49(25.7)$ \\
\hline 3 & $45(23.6)$ \\
\hline 4 & $29(15.2)$ \\
\hline 5 & $20(10.5)$ \\
\hline 6 & $8(4.2)$ \\
\hline$\geq 7$ & $9(4.7)$ \\
\hline \multicolumn{2}{|l|}{ M stage ${ }^{a}$} \\
\hline M0 & $8(4.2)$ \\
\hline M1a & $18(9.4)$ \\
\hline M1b & $16(8.4)$ \\
\hline M1c & $149(78.0)$ \\
\hline \multicolumn{2}{|l|}{ Visceral metastasis } \\
\hline No & $52(27.2)$ \\
\hline Yes & $139(72.8)$ \\
\hline \multicolumn{2}{|l|}{ CNS metastasis } \\
\hline No & $131(68.6)$ \\
\hline Yes & $60(31.4)$ \\
\hline \multicolumn{2}{|l|}{ Liver metastasis } \\
\hline No & $122(63.9)$ \\
\hline Yes & $69(36.1)$ \\
\hline \multicolumn{2}{|l|}{ Prior treatment regimens } \\
\hline Anti-CTLA-4 & $20(10.5)$ \\
\hline Anti-PD-1 & $10(5.2)$ \\
\hline $\mathrm{BRAFi} \pm \mathrm{MEKi}$ & $0(0.0)$ \\
\hline Chemotherapy & $21(11.0)$ \\
\hline Radiotherapy & $49(25.7)$ \\
\hline Adjuvant interferon & $59(30.9)$ \\
\hline \multicolumn{2}{|l|}{ Treatment } \\
\hline BRAFi monotherapy & $104(54.5)$ \\
\hline BRAFi + MEKi & $87(45.5)$ \\
\hline Dabrafenib & $22(11.5)$ \\
\hline Dabrafenib + trametinib & $47(24.6)$ \\
\hline Encorafenib & $3(1.6)$ \\
\hline Encorafenib + binimetinib & $14(7.3)$ \\
\hline Vemurafenib & $79(41.4)$ \\
\hline Vemurafenib + cobimetinib & $26(13.6)$ \\
\hline
\end{tabular}


Table 1 (continued)

\begin{tabular}{ll}
\hline Characteristic & $N(\%)$ \\
\hline Line of treatment & \\
First line & $116(60.7)$ \\
Second line & $57(29.8)$ \\
Third line & $11(5.8)$ \\
Fourth line & $7(3.7)$ \\
\hline
\end{tabular}

${ }^{a}$ American Joint Committee on Cancer staging guideline for melanoma from 2009

$B R A F$ B-Raf proto-oncogene, BRAFi BRAF inhibitor, $C N S$ central nervous system, $C T L A-4$ cytotoxic T-lymphocyte-associated protein 4, $M E K$ mitogen-activated protein kinase kinase, MEKi MEK inhibitor, $P D-1$ programmed cell death protein-1

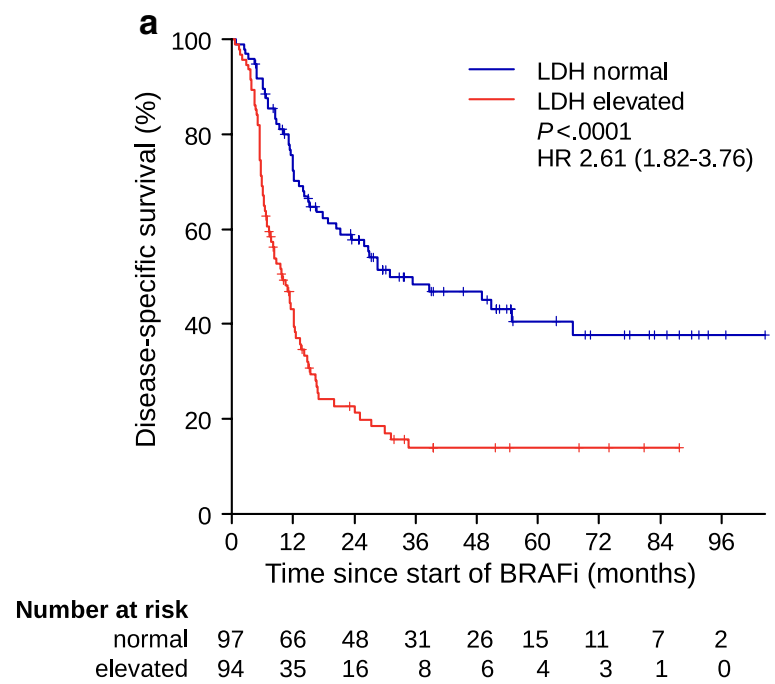

concentrations of $\operatorname{LDH}(P=0.016)$ and $\mathrm{S} 100 \mathrm{~B}(P<0.0001)$ significantly increased.

\subsection{Additional Prognostic Impact of LDH and S100B after Radiologically Confirmed Disease Progression}

DSSaP (timepoint of radiologically confirmed PD served as timepoint 0 ) was analyzed to assess the prognostic significance of LDH and S100B levels at the timepoint of PD in terms of further disease course after radiologically confirmed PD. No significant difference in DSSaP was observed between normal and elevated LDH (HR 1.48 [95\% CI 0.97-2.26]; $P=0.067$ ) (Fig. 4a), whereas patients with

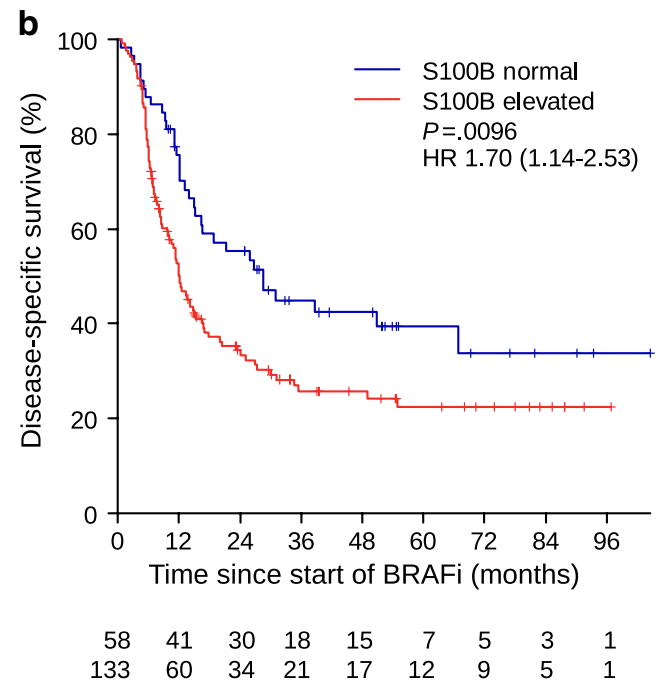

Fig. 1 Disease-specific survival according to a LDH and b S100B serum concentrations at baseline. BRAFi BRAF inhibitor therapy, $H R$ hazard ratio, $L D H$ lactate dehydrogenase, $P$ p-value

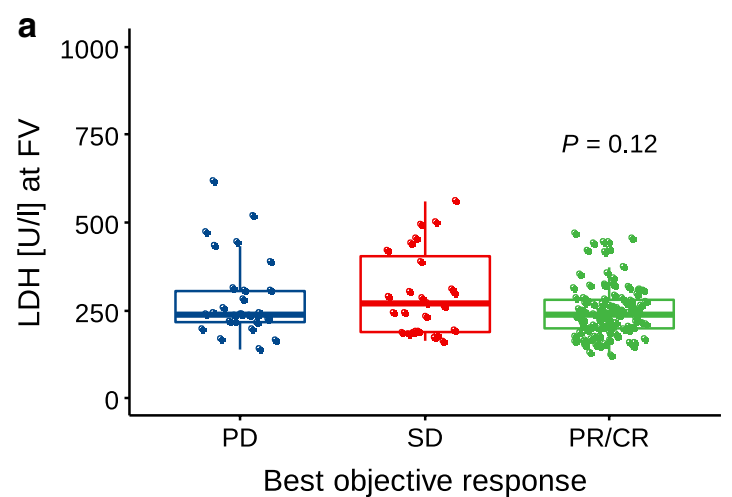

Fig. 2 Association of best objective response and $\mathbf{a}$ LDH and $\mathbf{b}$ S100B serum concentrations at first follow-up visit (FV) in $n=173$ patients (PD: $n=29$, SD: $n=27$, PR/CR: $n=117$ ). Statistical differences were calculated by unpaired two-sided Wilcoxon test com-

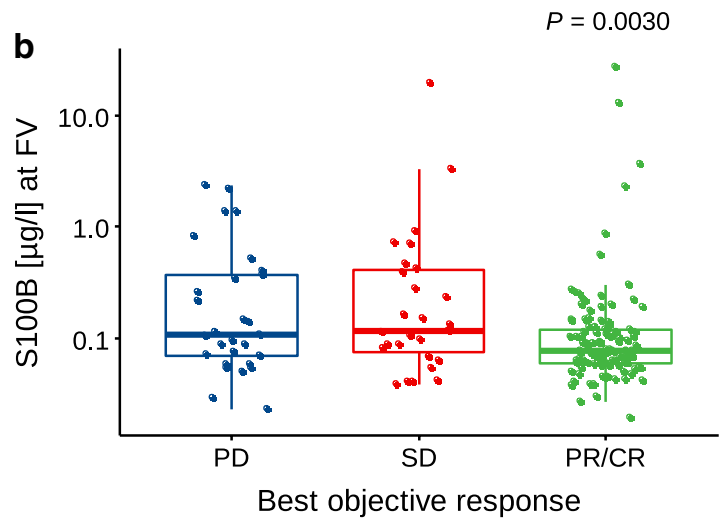

paring biomarker levels in patients with $\mathrm{PR} / \mathrm{CR}$ as best objective response compared with $\mathrm{PD} / \mathrm{SD}$. $C R$ complete response, $F V$ first visit, $L D H$ lactate dehydrogenase, $P$ p-value, $P D$ progressive disease, $P R$ partial response, $S D$ stable disease 


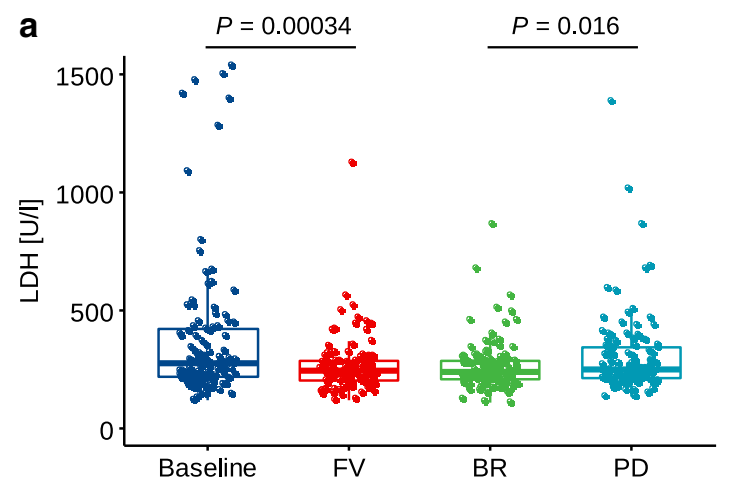

Fig. 3 a LDH and b S100B serum concentrations at baseline, first follow-up visit, staging examination showing the best objective response, and at time point of progression in patients who finally showed progressive disease $(n=121)$. Statistical differences were

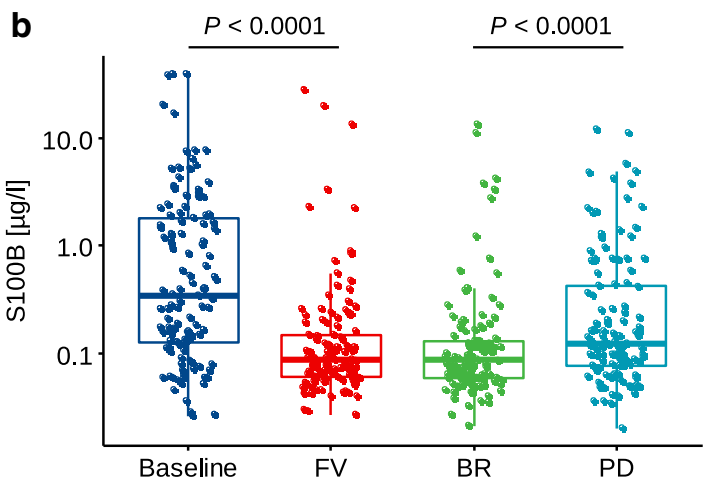

calculated by paired two-sided Wilcoxon test. $B R$ best response, $F V$ first visit, $L D H$ lactate dehydrogenase, $P$ p-value, $P D$ progressive disease
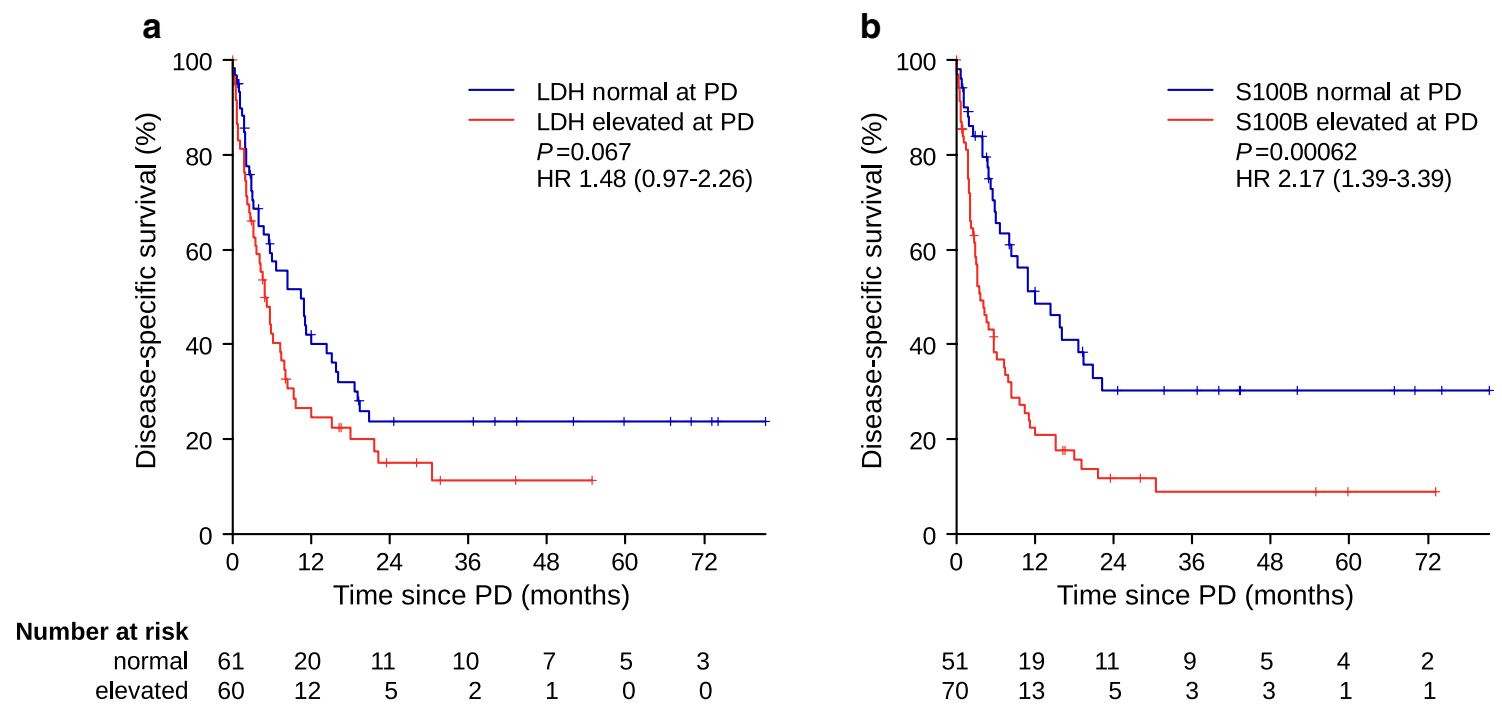

Fig. 4 Disease-specific survival beyond progressive disease according to $\mathbf{a} \mathrm{LDH}$ and $\mathbf{b} \mathrm{S} 100 \mathrm{~B}$ serum concentrations at time point of progression. $H R$ hazard ratio, $L D H$ lactate dehydrogenase, $P$ p-value, $P D$ progressive disease

elevated S100B at radiologically confirmed PD had significantly impaired DSSaP (HR 2.17 [95\% CI 1.39-3.39]; $P=$ 0.00062) (Fig. 4b). Multivariable Cox regression accounting for LDH and S100B at PD as well as number of metastatic sites and occurrence of new brain metastasis during BRAFi (model 1) treatment confirmed the independent prognostic significance of S100B measured at PD (Table 2). A second multivariable model (model 2) also included the baseline levels of LDH and S100B and showed comparable results.

The most intriguing question was whether the serum biomarkers could predict clinical progression some time before radiologic imaging. Interestingly, S100B stratified patients significantly (HR 1.93 [95\% CI 1.26-2.95]; $P=0.0024$ ) when it was measured as early as 56 days (IQR 41-83) before radiologically confirmed PD (Fig. 5a), whereas LDH did not (HR 1.35 [95\% CI 0.89-2.06]; $P=0.16$ ) (Fig. 5b).

\section{Discussion}

Our study shows that repeated measurement of serum LDH and $\mathrm{S} 100 \mathrm{~B}$ is useful to complement the close follow-up of patients receiving BRAFi. Elevated baseline LDH and baseline S100B were significantly associated with impaired DSS. The finding concerning LDH is in accordance with several previous studies showing the prognostic significance of LDH [1-3]. However, whether baseline S100B, which is widely used throughout Europe in addition to LDH because of its specificity, is also a prognostic factor for impaired DSS 
Table 2 Multivariate Cox regression analysis of disease-specific survival after progression according to risk factors

\begin{tabular}{|c|c|c|c|c|}
\hline \multirow[t]{2}{*}{ Risk factor } & \multicolumn{2}{|l|}{ Model $1(n=119)$} & \multicolumn{2}{|l|}{ Model $2(n=119)$} \\
\hline & HR $(95 \%$ CI $)$ & $p$ value & HR (95\% CI) & $p$ value \\
\hline $\mathrm{S} 100 \mathrm{~B}$ at $\mathrm{PD}$ & & 0.00045 & & 0.00030 \\
\hline Normal & 1 & & 1 & \\
\hline Elevated & $2.60(1.53-4.44)$ & & $2.75(1.59-4.77)$ & \\
\hline S100B at baseline & & & & 0.69 \\
\hline Normal & & & 1 & \\
\hline Elevated & & & $0.88(0.46-1.67)$ & \\
\hline $\mathrm{LDH}$ at PD & & 0.74 & & 0.49 \\
\hline Normal & 1 & & 1 & \\
\hline Elevated & $0.92(0.56-1.51)$ & & $0.84(0.51-1.38)$ & \\
\hline LDH at baseline & & & & 0.0098 \\
\hline Normal & & & 1 & \\
\hline Elevated & & & $1.97(1.18-3.30)$ & \\
\hline Metastatic sites & & 0.0021 & & 0.0033 \\
\hline $1-3$ & 1 & & 1 & \\
\hline$\geq 4$ & $2.03(1.29-3.19)$ & & $2.00(1.26-3.17)$ & \\
\hline New brain metastasis & & 0.24 & & 0.28 \\
\hline No & 1 & & 1 & \\
\hline Yes & $1.31(0.83-2.06)$ & & $1.28(0.81-2.03)$ & \\
\hline
\end{tabular}

Two cases were excluded because information was missing for one or more of the analyzed factors

$C I$ confidence interval, $H R$ hazard ratio, $L D H$ lactate dehydrogenase, $P D$ progressive disease, $S 100 B$ S100 calcium-binding protein B

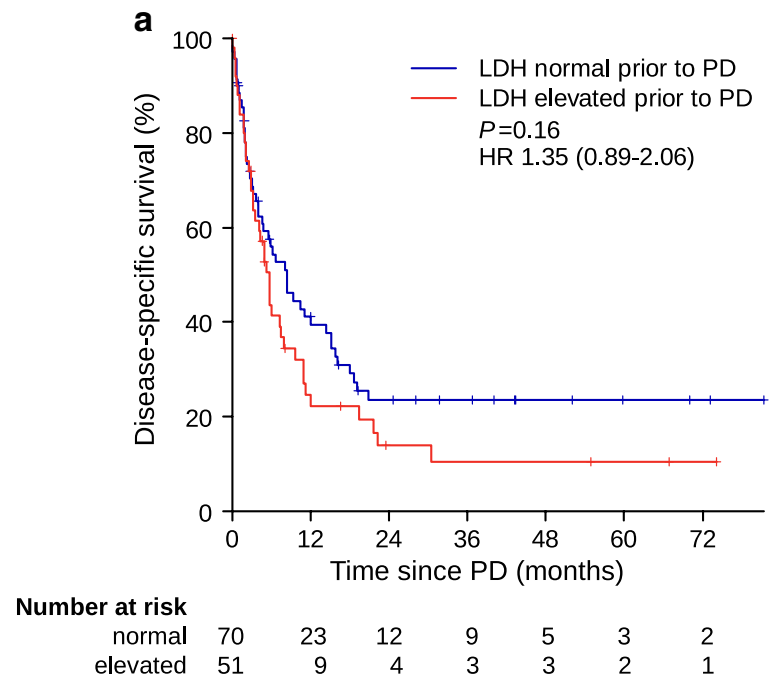

Fig. 5 Disease-specific survival beyond progressive disease according to a LDH and $\mathbf{b} \mathrm{S} 100 \mathrm{~B}$ serum concentrations prior to radiologically proven progression. Median time points of LDH and S100B measurements were performed 56 days (IQR 42-84) and 56 days

in patients treated with BRAFi was previously unknown. S100B is probably the best studied serum biomarker for melanoma and has been shown to be more sensitive than LDH in locoregionally metastasized stage III melanoma [17].

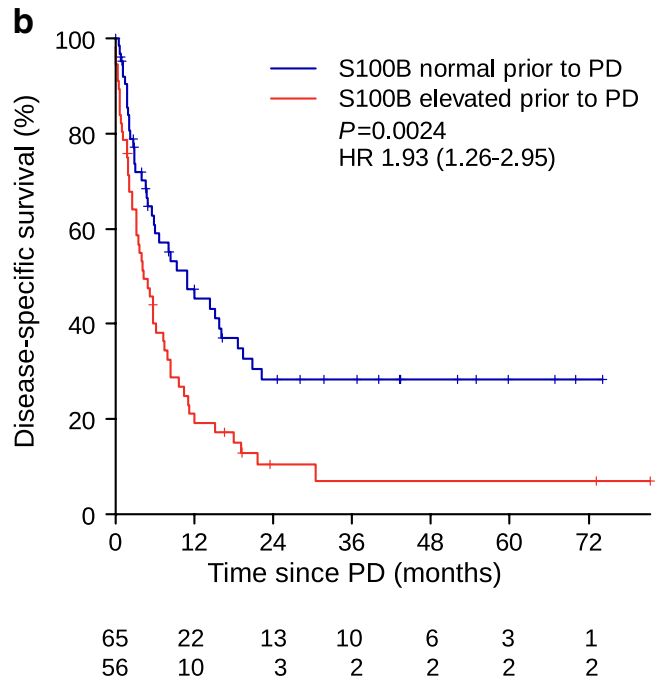

(IQR 41-83) prior to disease progression, respectively. IQR interquartile range, $H R$ hazard ratio, $L D H$ lactate dehydrogenase, $P$ p-value, $P D$ progressive disease

S100B has also been extensively studied in stage IV melanoma, and elevated levels were consistently associated with impaired survival, comparable to LDH [18, 19]. Our group has previously shown that elevated baseline S100B and LDH 
correlated with impaired survival of patients receiving ICIs [20]. It seems advisable to implement the measurement of baseline LDH in conjunction with S100B before commencing treatment with BRAFi and MEKi.

This study is the first to show that serum S100B measured during therapy with BRAFi at the time of the first FV during treatment correlates with BR. The median duration between start of treatment and S100B measurement at FV was 41 days (IQR 24-57). The median time point of FV was 26 days before median BR, which was 67 days after the initiation of targeted therapy. Thus, early measurement of S100B is capable of amending radiologic staging examinations to predict BR within the first weeks of therapy. Interestingly, LDH serum levels did not differ significantly between responders and nonresponders, indicating lower specificity than S100B. LDH and S100B levels measured at baseline and during treatment at FV, BR, and PD in patients who subsequently developed PD correlated with the course of the disease. Serum LDH and S100B levels significantly decreased between baseline and FV before increasing significantly between BR and PD. These findings are in accordance with the results obtained by a small cohort comprising 18 patients treated with vemurafenib [15]. The only other study including S100B and LDH measurements over time during therapy with BRAFi also found convincing accuracy of S100B and LDH in predicting response but reported a low accuracy of only $30 \%$ in predicting progression [14]. The authors of that study summarized that measuring these serum biomarkers was not an adequate alternative to imaging examinations. And indeed, also in our data, the accuracy of S100B and LDH correlating with PD in terms of elevated levels above the upper limit of normal was rather modest (S100B 70/121 patients [57.9\%]; LDH 60/121 [49.6\%]). Of note, 27 additional patients had increasing albeit normal serum S100B levels. When both patients with elevated S100B at progression and those with increasing albeit normal S100B at progression were combined, sensitivity increased up to $80.2 \%$.

Importantly, in patients with radiologically ascertained PD, S100B could discriminate these patients in terms of DSSaP (median DSSaP 12.0 [IQR 8.0-22.1] vs. 3.6 [IQR 2.8-7.2] months, respectively). As demonstrated in multivariable analysis, this result was independent of baseline biomarker levels, number of metastatic sites, and occurrence of new brain metastasis during BRAFi therapy, which constitutes a common confounder of serum biomarker levels in otherwise responding patients harboring low total tumor burden. LDH at progression was not significantly prognostic for DSSaP, either in univariate or in multivariate analysis. These results for LDH at progression are only partly in line with results from a study by Chan et al. [21], who found elevated LDH at progression was associated with impaired survival after progression in univariable but not in multivariable analysis. Compared with LDH, which did not yield significant results when analyzed at or before PD, S100B identified patients at considerably higher risk of significantly impaired survival at a median of 56 (IQR 41-83) days before radiologically confirmed $\mathrm{PD}$. These findings underline the higher sensitivity of S100B compared with LDH to predict metastasis, even under targeted therapy.

Previous studies showed a strong correlation between both the pattern and the intensity of S100B expression in melanoma tissue and in serum [22]. A minority, but still a significant number of up to $8 \%$ of melanoma tissue samples are S100 negative, partly explaining cases with significant tumor burden but normal S100B serum levels [23]. However, de Vries et al. [24] found a consistent and high percentage of S100 immunoreactive cells in tissue samples of primary melanomas, locoregional soft tissue metastases, lymph node metastases, and visceral metastases from the lung, liver, and brain. Other authors also demonstrated that more than $90 \%$ of melanoma or melanoma metastases show S100 staining and, in contrast to differentiation antigens and cancer testis antigens, S100 was not lost during metastasis $[25,26]$. Despite its sensitivity and specificity for predicting visceral, brain, and-in part-lymph node metastasis, S100B was reported to have modest sensitivity for local recurrence or skin metastasis $[27,28]$.

The strengths of the present study include the relatively large number of patients, long follow-up, and analysis of S100B and LDH levels at five distinct timepoints. We report for the first time that $\mathrm{S} 100 \mathrm{~B}$ but not $\mathrm{LDH}$ can discriminate patients with impaired survival from patients with favorable survival after progression to BRAFi even before PD is detected by radiologic imaging. This is an unexpected yet important finding that highlights the clinical relevance of serial S100B measurements during targeted therapy. In contrast to ICI, there is a substantial risk of sudden progression despite initial response to targeted therapy with BRAFi and MEKi. In the recently published 5-year outcomes of patients treated with dabrafenib and trametinib, only $16 \%$ who had initially achieved a PR as BR were still progression-free after 5 years, and nearly three-quarters of these progressions occurred within the first 2 years of treatment [9]. Therefore, early detection of disease progression with a combination of close radiologic imaging and serial S100B measurements are particularly important for patients treated with BRAFi and MEKi.

Our study has a few limitations. First, the data were collected retrospectively and derive from both patients treated in the context of clinical trials and those treated in routine clinical practice. However, all patients included in this study were consecutively treated at our institution and were not arbitrarily chosen. Moreover, the timepoints of the laboratory tests were non-standardized because of distinct on-therapy schedules that were used outside of clinical trials. We cannot exclude a resulting bias of our results. Nevertheless, 
the comparability of the results for DSSaP between the two distinct timepoints prior to PD on the one hand and radiologically proven PD on the other hand validates these results.

In summary, our study shows the advantages of melanoma serum biomarker S100B compared with LDH in repeated measurements during targeted therapy of metastatic melanoma with BRAFi. While LDH was more strongly associated with DSS at baseline than was S100B, the latter outperformed LDH in terms of association with BR and PD. Serial measurements of S100B were helpful in predicting PD and DSSaP, even several weeks before PD was radiologically confirmed. Therefore, it seems appropriate to recommend the implementation of serial S100B measurements, at least monthly, during targeted therapy.

Supplementary Information The online version contains supplementary material available at https://doi.org/10.1007/s11523-021-00792-8.

Acknowledgements The authors thank the melanoma unit team for their passionate patient care and support in data collection.

\section{Declarations}

Funding No external funding was used in the preparation of this manuscript. Open Access funding enabled and organized by Projekt DEAL.

Conflict of interest Maximilian Gassenmaier has served on an advisory board for and received grants from Novartis outside the submitted work. Andrea Forschner has received speaker's honoraria from BMS, MSD, Roche, and Novartis; served on an advisory board for Roche and Novartis; and received travel support from Roche, Novartis, and BMS outside the submitted work. Ulrike Leiter has served on an advisory board or as a consultant for and received honoraria from MSD, Novartis, and Roche outside the submitted work. Clause Garbe has received grants and personal fees from BMS, Roche, and Novartis and personal fees from Amgen, MSD, and Philogen outside the submitted work. Thomas K. Eigentler has served on an advisory board or as a consultant for Philogen, BMS, Novartis, Roche, and Sanofi outside the submitted work. Nikolaus B. Wagner has served on an advisory board for Sanofi outside the submitted work. Max M. Lenders and Benjamin Weide have no conflicts of interest that are directly relevant to the content of this article.

Ethics approval Approval to conduct this study was obtained from the ethics committee of the Medical Faculty of University Tübingen (project no. 257/2018BO2).

Consent to participate Not applicable.

Consent for publication Not applicable.

Availability of data and material The datasets generated and/or analyzed during the current study are available from the corresponding author on reasonable request.

Code availability The statistical codes used during the current study are available from the corresponding author on reasonable request.

Author contributions Study concept and design: NBW. Data collection and statistical analysis: MG, MML, TKE, NBW. Writing and criti- cal revision of the draft: MG, MML, AF, UL, BW, CG, TKE, NBW. Supervision: NBW.

Open Access This article is licensed under a Creative Commons Attribution-NonCommercial 4.0 International License, which permits any non-commercial use, sharing, adaptation, distribution and reproduction in any medium or format, as long as you give appropriate credit to the original author(s) and the source, provide a link to the Creative Commons licence, and indicate if changes were made. The images or other third party material in this article are included in the article's Creative Commons licence, unless indicated otherwise in a credit line to the material. If material is not included in the article's Creative Commons licence and your intended use is not permitted by statutory regulation or exceeds the permitted use, you will need to obtain permission directly from the copyright holder. To view a copy of this licence, visit http://creativecommons.org/licenses/by-nc/4.0/.

\section{References}

1. Larkin J, Del Vecchio M, Ascierto PA, Krajsova I, Schachter J, Neyns B, et al. Vemurafenib in patients with BRAF(V600) mutated metastatic melanoma: an open-label, multicentre, safety study. Lancet Oncol. 2014;15(4):436-44.

2. Ascierto PA, McArthur GA, Dreno B, Atkinson V, Liszkay G, Di Giacomo AM, et al. Cobimetinib combined with vemurafenib in advanced BRAF(V600)-mutant melanoma (coBRIM): updated efficacy results from a randomised, double-blind, phase 3 trial. Lancet Oncol. 2016;17(9):1248-60.

3. Long GV, Flaherty KT, Stroyakovskiy D, Gogas H, Levchenko E, de Braud F, et al. Dabrafenib plus trametinib versus dabrafenib monotherapy in patients with metastatic BRAF V600E/K-mutant melanoma: long-term survival and safety analysis of a phase 3 study. Ann Oncol. 2017;28(7):1631-9.

4. Larkin J, Ascierto PA, Dréno B, Atkinson V, Liszkay G, Maio M, et al. Combined vemurafenib and cobimetinib in BRAF-mutated melanoma. N Engl J Med. 2014;371(20):1867-76.

5. Robert C, Karaszewska B, Schachter J, Rutkowski P, Mackiewicz A, Stroiakovski D, et al. Improved overall survival in melanoma with combined dabrafenib and trametinib. N Engl J Med. 2015;372(1):30-9.

6. Long GV, Stroyakovskiy D, Gogas H, Levchenko E, de Braud F, Larkin J, et al. Combined BRAF and MEK inhibition versus BRAF inhibition alone in melanoma. $\mathrm{N}$ Engl $\mathrm{J}$ Med. 2014;371(20):1877-88

7. Van Allen EM, Wagle N, Sucker A, Treacy DJ, Johannessen CM, Goetz EM, et al. The genetic landscape of clinical resistance to RAF inhibition in metastatic melanoma. Cancer Discov. 2014:4(1):94-109.

8. Larkin J, Chiarion-Sileni V, Gonzalez R, Grob JJ, Rutkowski $\mathrm{P}$, Lao CD, et al. Five-year survival with combined nivolumab and ipilimumab in advanced melanoma. $\mathrm{N}$ Engl $\mathrm{J}$ Med. 2019;381(16): 1535-46.

9. Robert C, Grob JJ, Stroyakovskiy D, Karaszewska B, Hauschild A, Levchenko E, et al. Five-year outcomes with dabrafenib plus trametinib in metastatic melanoma. $\mathrm{N}$ Engl $\mathrm{J}$ Med. 2019;381(7):626-36.

10. Long GV, Grob JJ, Nathan P, Ribas A, Robert C, Schadendorf $\mathrm{D}$, et al. Factors predictive of response, disease progression, and overall survival after dabrafenib and trametinib combination treatment: a pooled analysis of individual patient data from randomised trials. Lancet Oncol. 2016;17(12):1743-54.

11. Schadendorf D, Long GV, Stroiakovski D, Karaszewska B, Hauschild A, Levchenko E, et al. Three-year pooled analysis of 
factors associated with clinical outcomes across dabrafenib and trametinib combination therapy phase 3 randomised trials. Eur J Cancer. 2017;82:45-55.

12. Egberts F, Hitschler WN, Weichenthal M, Hauschild A. Prospective monitoring of adjuvant treatment in high-risk melanoma patients: lactate dehydrogenase and protein S-100B as indicators of relapse. Melanoma Res. 2009;19(1):31-5.

13. Egberts F, Kotthoff EM, Gerdes S, Egberts JH, Weichenthal M, Hauschild A. Comparative study of YKL-40, S-100B and $\mathrm{LDH}$ as monitoring tools for stage IV melanoma. Eur J Cancer. 2012;48(5):695-702.

14. Abusaif S, Jradi Z, Held L, Pflugfelder A, Weide B, Meier F, et al. S100B and lactate dehydrogenase as response and progression markers during treatment with vemurafenib in patients with advanced melanoma. Melanoma Res. 2013;23(5):396-401.

15. Sanmamed MF, Fernandez-Landazuri S, Rodriguez C, Lozano MD, Echeveste JI, Perez Gracia JL, et al. Relevance of MIA and S100 serum tumor markers to monitor BRAF inhibitor therapy in metastatic melanoma patients. Clin Chim Acta. 2014;429:168-74.

16. Eisenhauer EA, Therasse P, Bogaerts J, Schwartz LH, Sargent D, Ford R, et al. New response evaluation criteria in solid tumours: revised RECIST guideline (version 1.1). Eur J Cancer. 2009;45(2):228-47.

17. Wevers KP, Kruijff S, Speijers MJ, Bastiaannet E, Muller Kobold AC, Hoekstra HJ. S-100B: a stronger prognostic biomarker than LDH in stage IIIB-C melanoma. Ann Surg Oncol. 2013;20(8):2772-9.

18. Egberts F, Pollex A, Egberts JH, Kaehler KC, Weichenthal M, Hauschild A. Long-term survival analysis in metastatic melanoma: serum S100B is an independent prognostic marker and superior to LDH. Onkologie. 2008;31(7):380-4.

19. Weide B, Elsasser M, Buttner P, Pflugfelder A, Leiter U, Eigentler TK, et al. Serum markers lactate dehydrogenase and S100B predict independently disease outcome in melanoma patients with distant metastasis. Br J Cancer. 2012;107(3):422-8.

20. Wagner NB, Forschner A, Leiter U, Garbe C, Eigentler TK. $\mathrm{S} 100 \mathrm{~B}$ and LDH as early prognostic markers for response and overall survival in melanoma patients treated with anti-PD-1 or combined anti-PD- 1 plus anti-CTLA- 4 antibodies. Br J Cancer. 2018;119(3):339-46.

21. Chan MM, Haydu LE, Menzies AM, Azer MW, Klein O, Lyle $\mathrm{M}$, et al. The nature and management of metastatic melanoma after progression on BRAF inhibitors: effects of extended BRAF inhibition. Cancer. 2014;120(20):3142-53.

22. Banfalvi T, Udvarhelyi N, Orosz Z, Gergye M, Gilde K, Timar J. Heterogenous S-100B protein expression patterns in malignant melanoma and association with serum protein levels. Oncology. 2003;64(4):374-9.

23. Viray H, Bradley WR, Schalper KA, Rimm DL, Gould Rothberg BE. Marginal and joint distributions of S100, HMB-45, and Melan-A across a large series of cutaneous melanomas. Arch Pathol Lab Med. 2013;137(8):1063-73.

24. de Vries TJ, Smeets M, de Graaf R, Hou-Jensen K, Brocker EB, Renard N, et al. Expression of gp100, MART-1, tyrosinase, and S100 in paraffin-embedded primary melanomas and locoregional, lymph node, and visceral metastases: implications for diagnosis and immunotherapy. A study conducted by the EORTC Melanoma Cooperative Group. J Pathol. 2001;193(1):13-20.

25. Trefzer U, Hofmann M, Reinke S, Guo YJ, Audring H, Spagnoli $\mathrm{G}$, et al. Concordant loss of melanoma differentiation antigens in synchronous and asynchronous melanoma metastases: implications for immunotherapy. Melanoma Res. 2006;16(2):137-45.

26. Lim E, Browning J, MacGregor D, Davis ID, Cebon JS. Desmoplastic melanoma: comparison of expression of differentiation antigens and cancer testis antigens. Melanoma Res. 2006;16(4):347-55.

27. Seregni E, Massaron S, Martinetti A, Illeni MT, Rovini D, Belli F, et al. S100 protein serum levels in cutaneous malignant melanoma. Oncol Rep. 1998;5(3):601-4.

28. Kaskel P, Berking C, Sander S, Volkenandt M, Peter RU, Krahn G. S-100 protein in peripheral blood: a marker for melanoma metastases: a prospective 2-center study of 570 patients with melanoma. J Am Acad Dermatol. 1999;41(6):962-9. 